\title{
Bella ciao
}

\author{
di Marida Brignani
}

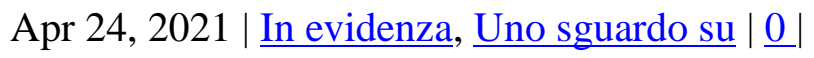
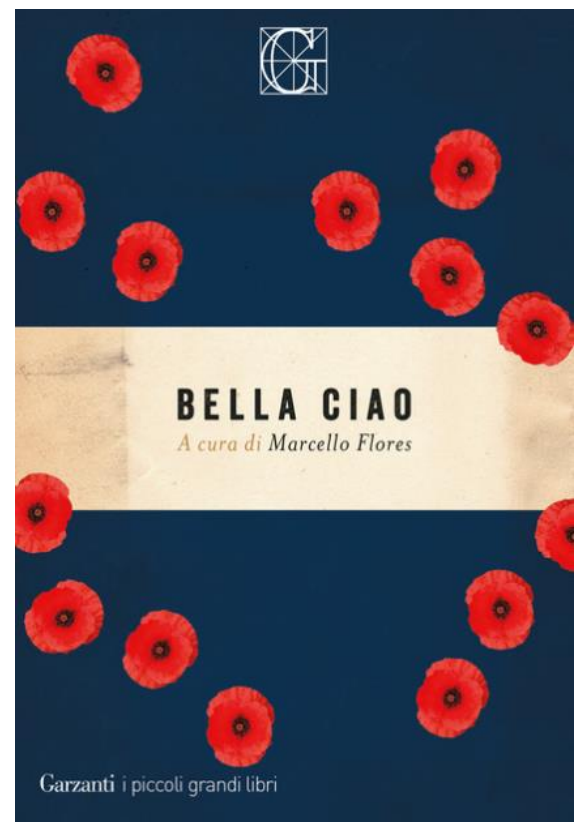

Copertina dal sito dell'editore

\section{Marcello Flores (a cura di) \\ Bella ciao, a cura di Marcello Flores \\ Garzanti, Milano, 2020, pp. 96.}

Nell'aprile 2020, a cura di Marcello Flores, viene pubblicato nella collana I Piccoli Grandi Libri Garzanti un nuovo studio dedicato a Bella ciao, il canto resistenziale italiano più famoso. Un libretto accattivante, di quelli che attraggono subito: breve, avvolto in una copertina nitida, stampato in formato minuscolo e leggero, piacevole da maneggiare e da leggere ovunque. Uno di quei piccoli libri che promettono di soddisfare piacevolmente una curiosità e offrono la certezza di trovarvi conoscenza. L'autore, con un linguaggio lineare e piano adatto anche agli studenti, ricompone e porta a sintesi numerosi studi precedenti - accuratamente citati per chi volesse approfondire - e propone una visione complessiva, ampia e aggiornata, dei tentativi di ricostruire la nascita, l'uso e la trasformazione dei significati di un canto italiano della Resistenza, oggi noto in tutto il mondo, aprendo nuovi spazi d'indagine e di studio sulla storia e le trasformazioni del pensiero, del linguaggio, della sensibilità politica e sociale del Novecento. E di riflessione sul presente.

L'interesse per Bella ciao e altri canti - popolari, politici, di guerra e di lavoro - si sviluppa a partire dai primi anni Sessanta, in un clima culturale che sta mutando e che guarda con nuovo interesse allo studio dell' antropologia e del folklore, mentre si ravviva l'attenzione sul fenomeno complessivo della Resistenza. Un ruolo fondamentale ha la pubblicazione del Nuovo canzoniere italiano (1962), sulla scia degli studi del gruppo torinese Cantacronache, seguìto, nel 1964, da uno spettacolo lanciato al Festival dei Due Mondi di Spoleto, intitolato proprio Bella ciao. L'esibizione genera una lunga scia di polemiche per l'esecuzione in versione integrale di un canto della Prima 
guerra mondiale - O Gorizia tu sei maledetta - ritenuto oltraggioso per le forze armate. Sia il successo dello spettacolo, sia la visibilità mediatica innescata dalle polemiche, contribuiscono a divulgare la conoscenza del canto, proposto in apertura e in chiusura della performance teatrale, fino a fargli valicare i confini nazionali.

Seguono nuovi studi e ricerche per ricostruirne la genesi, non senza contraddizioni e colpi di scena: da chi ne individua l'origine in un canto delle mondine, a chi sostiene - poi smentito - che non fu mai utilizzato durante la Resistenza, fino alle improvvise e plurime dichiarazioni di paternità di sedicenti autori. Ma la versione prima e originaria rimane per il momento sconosciuta. Il canto pare accogliere molte influenze diverse: vi si leggono echi di musiche popolari dell'Ottocento, di canti che accompagnavano le lunghe marce dei fanti nella Grande Guerra, di canti politici, della risaia e della Resistenza, senza escludere il tema dell'amore.

Grazie all'interpretazione di molti artisti nazionali e internazionali, a partire da Ives Montand, il canto entra nel repertorio della musica leggera e commerciale. Basta consultare oggi il canale You tube per rendersi conto delle sue numerosissime traduzioni e interpretazioni in tutte le lingue del mondo. Altrettanto significativa la sua comparsa in spettacoli, film e serie televisive, fra le quali non si può tacere la recente serie spagnola La casa de Papel (La casa di carta, proposta anche in Italia), il cui enorme successo ha contribuito in maniera rilevante, secondo Flores, a diffonderne la conoscenza a livello planetario. Ciò che emerge a ogni nuovo approfondimento è come la sua natura non violenta e trasversale consenta di ampliarne i significati, dalla esclusiva simbologia della lotta al nazifascismo ai valori universali della pace, della giustizia e della libertà, di cui diventa l'inno. A ogni significativo cambiamento del contesto sociopolitico internazionale il canto è riletto e riutilizzato con nuove sfumature di significato. Emblematiche sono le molte circostanze in cui è intonato, che consentono di attraversare un secolo di storia: se scontata (ancorché controversa) è la sua esecuzione nelle piazze in occasione della Festa di Liberazione, meno lo è l'ultimo saluto alle esequie di celebri politici e intellettuali e, ancor più sorprendente, la sua recente trasformazione in inno della resistenza armata delle donne curde di Kobane mentre combattono lo Stato islamico.

Ma per quale motivo proprio Bella ciao incontra questo favore e non altri canti resistenziali, originariamente più conosciuti e non meno incisivi? Per una serie di ragioni, sia di forma che di sostanza. È innanzitutto un canto meno divisivo e ideologico di altri e ha minori riferimenti partitici: a partire dagli anni Sessanta, in concomitanza con la ricerca di un clima di maggiore concordia, molte delle diverse anime della sinistra vi si possono riconoscere. Dopo l'interpretazione di Montand, il canto amplia i propri confini e da simbolo della resistenza italiana diviene un filo che lega le resistenze europee. $\grave{E}$ infatti un canto che individua precisamente il proprio nemico esclusivamente nello straniero usurpatore, tema condivisibile praticamente da tutti. Non è intriso di retorica, ma è un canto d'amore, di fiducia e di speranza. Più che la resistenza, canta con passione $\mathrm{i}$ suoi risultati. Grande importanza ha poi la sua musicalità: semplice, incisiva ma non banale e abbastanza moderna da cogliere la sensibilità delle generazioni post-belliche.

Bella ciao può essere un malinconico canto solista accompagnato da un coro, ma anche un canto corale ritmato dal battito delle mani. Pochi semplici accorgimenti, anche spontanei, ne possono facilmente adattare il ritmo a un incedere più incisivo e incalzante, o gioioso. La partitura musicale regge senza problemi la traduzione del testo in qualsiasi lingua e le due parole che compongono il titolo e il ritornello, spesso lasciati in lingua originale, sono fra i termini italiani più noti e comprensibili in ogni parte del mondo. Un canto dunque che, partito dalle campagne italiane, senza essere snaturato nella propria intenzione comunicativa originale, ha trovato voce per diffondersi autonomamente e con sfumature diverse in tutto il mondo. Se ne trovano moltissimi arrangiamenti e interpretazioni di grande suggestione che attraversano ormai tutti i confini. 
Un anno è trascorso dalla pubblicazione del libro, uscito in pieno lockdown e a librerie chiuse, e nessuno può dimenticare il modo in cui abbiamo vissuto quel periodo. Serrati nelle nostre case, le strade e le piazze deserte, attoniti, sentivamo mancare l'aria. Lo sentivano fisicamente e in modo drammatico i contagiati, lo sentivano metaforicamente tutti gli altri. L'aria che mancava non era solo l'ossigeno che scarseggiava nelle terapie intensive, era anche il contatto con i propri cari, con gli amici e i colleghi. Erano i luoghi soliti dell'incontro: la strada, la piazza, il caffè sotto casa, il mercato e i negozi di quartiere, i luoghi dello studio, del lavoro, della cultura e dell'intrattenimento. Erano i gesti quotidiani più innocui divenuti improvvisamente pericolosi e addirittura vietati. Erano i viaggi e le mete perdute. Era la primavera che esplodeva senza di noi: non vista, non assaporata, non attraversata. Osservavamo il mondo da una molteplicità di finestre: i vetri e i balconi delle nostre case, la televisione, i piccoli e grandi schermi di pc, tablet e smartphone. Ne percepivamo tutta l'importanza per non sentirci isolati e, al tempo stesso, ne comprendevamo l'inadeguatezza a restituirci la pienezza delle nostre relazioni. Nella nostra solitudine iperconnessa, abbiamo scoperto, improvvisamente e drammaticamente, che la libertà non è solo un concetto astratto che esprime un diritto fondamentale degli uomini, declinato in molteplici e irrinunciabili forme e casistiche reali (al valore delle quali troppo spesso non prestiamo più la dovuta attenzione, abituati a sentirle parte della nostra quotidianità da 75 anni). Abbiamo toccato con mano che la libertà è anche l'insieme di tanti piccoli gesti, delle abitudini più semplici, spontanee e naturali della nostra vita. E che in un attimo la possiamo perdere.

Un anno fa la reazione spontanea è stata generosa, vitale, passionale. Nel tempo sospeso e metafisico delle nostre città ha fatto irruzione urlata, e soprattutto cantata, la voglia di riscatto, di ripresa, di solidarietà. Striscioni e bandiere alle finestre, flash mob di canti e scenografie luminose dai balconi e migliaia di video che rimbalzavano sui social per esorcizzare il dramma assoluto di quanto stava accadendo. Stupore e ironia, dolcezza e nostalgia, coraggio, retorica, amore, bellezza, arte, speranza... e tanta, tanta musica. Una colonna sonora spontanea che prendeva corpo giorno dopo giorno adattandosi alle tante sfumature di un dramma destinato a segnare in modo indelebile la storia di questo secolo e che si andava consumando a livello planetario sotto i nostri occhi, mai vissuto così coralmente in tempo reale. E la musica a sottolinearne di volta in volta l'intensità in una altalenante gamma di umori e sentimenti. Orgoglio nazionale, riscatto, fiducia nella propria forza con le frecce tricolori e le immagini delle bellezze del patrio suolo sottolineate dall'inno nazionale e dal Nessun dorma della pucciniana Turandot; il sentimento struggente di nostalgia e il desiderio di ritrovare la perduta libertà nel coro del Nabucco e ancora, di fronte allo sconvolgente numero delle perdite umane che sembrava non vedere fine e al sacrificio generoso di tanti operatori sanitari, le note struggenti e sublimi dei più straordinari capolavori della musica classica, non solo religiosa, suonate e disperse nel vento dai tetti di luoghi altamente simbolici. Compositori, musicisti, interpreti di grande fama che mettevano a disposizione la propria arte, ma anche canti e musiche della tradizione napoletana, musica leggera, pop e cantautoriale, brani gioiosamente vitali della tradizione popolare, fino al mitico Azzurro, assurto al ruolo di inno nazional-popolare che, rimbalzando di balcone in balcone, attraversava la penisola.

E Bella ciao, suonata e cantata dai balconi e dalle finestre. Ma se potevamo facilmente immaginare che diventasse ancora una volta momento di aggregazione nella piazza - questa volta virtuale - del web in occasione del 25 Aprile, sorprendente ed emozionante è stato scoprire con quanta spontanea partecipazione il canto avesse trovato una ennesima, nuova dimensione e un nuovo significato. Improvvisamente, da un capo all'altro del mondo, si è sentito rimbalzare quale dedica e incoraggiamento a un'Italia sofferente, attonita e un po' arruffata che cercava la forza di resistere a un nuovo nemico subdolo e invisibile. In italiano, o tradotta nelle varie lingue e arricchita delle diverse sonorità culturali, Bella ciao veniva "restituita" sotto forma di omaggio, incoraggiamento, partecipazione e speranza all'Italia. Arricchiva ancora, e non certo in modo definitivo, le sfumature del proprio significato di inno alla libertà, all'amore, alla solidarietà, con la voglia di diventare «una 
promessa, anzi un patto civile necessario per costruire l'avvenire. Senza rimpianti per il passato e con una grande nostalgia per il futuro»[1].

Note:

[1] David Bidussa, 24 aprile 2020 (https://www.joimag.it/bella-ciao-la-canzone-per-pensare-adomani/) 